\title{
Isolated xanthogranuloma of pelvico-ureteric junction: is this beginning of xanthogranulomatous pyelonephritis? - a case report
}

\begin{abstract}
Xanthogranulomatous Pyelonephritis (XGP) is a rare condition characterised by chronic granulomatous inflammation of renal tissue. ${ }^{1,2}$ This can be due to obstruction, urinary tract infection and renal calculi. ${ }^{1-5}$ The exact pathophysiology and progression of this condition is poorly understood, but altered immune response, changes in lipid metabolism, disturbances in leukocyte function, lymphatic obstruction, venous occlusion/haemorrhage, malnutrition, arterial insufficiency and necrosis of pericalyceal fat. ${ }^{2,4}$ Its management with surgical intervention in the form of nephrectomy, partial or complete, is the most definitive management option of this condition. ${ }^{1}$ We report the case of a woman with isolated xanthogranuloma obstructing the pelviureteric junction. We propose this as the initial pathophysiological process of Xanthogranuloma pyelonephritis.
\end{abstract}

Keywords: isolated, xanthogranulomatous, pyelonephritis, pelvico-ureteric junction, urology
Volume 4 Issue 4 - 2017

\author{
Devang J Desai ${ }^{1,2,3}$, Kevin Su ${ }^{1,2}$, Ross Smith ${ }^{1,2}$, \\ John Preston ${ }^{1,2}$ \\ 'Department of Urology, Princess Alexandra Hospital,Australia \\ 2University of Queensland, Australia \\ ${ }^{3}$ Toowoomba Urology, Toowoomba, Australia
}

Correspondence: Devang Desai, Department of Urology, Princess Alexandra Hospital, 199 Ipswich road,Woolloongabba, Brisbane, Queensland, Australia 4102, Email ddesai@ toowoombaurology.com.au

Received: January 09, 2017 | Published: April 19, 2017
Abbreviations: XGP, xanthogranulomatous pyelonephritis; LMO, local medical officer; UTI, urinary tract infection; PUJ, pyrloureteric junction; $\mathrm{CT}$, computed tomography

\section{Case}

A 74-year-old female presented to hospital with a two-day history of right flank pain and fever referred by her local medical officer (LMO) who initially diagnosed her with a Klebsiella urinary tract infection (UTI) and managed empirically with cephalexin and urinary alkalinisers. This patient had a medical background of Gastro-oesophageal Reflux Disease, Migraines, Diverticulitis, hypothyroidism, Hypertension, Aortic valve dysfunction, Type 2 Diabetes Mellitus, Dyslipidaemia, Asthma and bilateral congenital deafness. Medications at presentation were amitriptyline, aspirin, esomeprazole, telmisartan, thyroxine, salbutamol, metformin, fluticasone/salmeterol, cyproheptadine, verapamil and mebeverine.

She also has a previous surgical history of appendectomy at age four. In the emergency department, she was found to be febrile to $38.7^{\circ} \mathrm{C}$, blood pressure of $156 / 59, \mathrm{O}_{2}$ saturation of $91 \%$ on room air, blood sugar level of $10.9 \mathrm{mmol} / \mathrm{L}$, respiratory rate of $20 / \mathrm{min}$, and pulse rate of $90 / \mathrm{min}$. Examination revealed the presence of bowel sounds, moderate to severe pain and tender right flank and iliac fossa with no guarding, rebound and the presence of bowel sounds. Urine microscopy subsequently demonstrated pyuria and Morganella Morganii was cultured. Blood cultures were negative. Concerns for an acutely obstructed infected right kidney were raised when it was noted that her creatinine was $84 \mathrm{umol} / \mathrm{L}$ and eGFR was 56 . A non-contrast CT was performed (see image) showing right peri-nephric stranding, dilatation of the right pelvicalyceal system to the pyrloureteric junction (PUJ) and no evidence of calculus or other aetiology of the obstruction. There was periureteric fat stranding along, atypical for PUJ obstruction.
She was transferred to a tertiary referral hospital; renal function had further deteriorated to a Creatinine of $99 \mathrm{umol} / \mathrm{L}$ and an eGFR of 49 . On arrival, a nephrostomy tube was inserted. She went on to develop urosepsis. This was treated with intravenous antibiotics tailored as per culture results. Due to the proximity of the inflammatory process to the duodenum, upper endoscopy was performed with no abnormality seen. Further investigations involved nephrostogram and ureteroscopy. Findings were that of urothelial thickening and stricturing of the PUJ raising the possibility of a malignant stricture. There was fat stranding that extended to the duodenum but no associated duodenal thickening to suggest tethering or duodenal pathology. A biopsy was taken and a ureteric stent placed. Definitive management involved an open right PUJ exploration, frozen section biopsy and repair. Intraoperatively, it was noted that the right PUJ was adhered to the duodenum and some thick fibrotic tissue involving right PUJ. This area was excised and sent for frozen section, histology of the sample revealing Xanthogranulomatous inflammation with extensive fibrosis. A dismembered pyeloplasty was performed and she had an uneventful recovery with a 3-month follow-up.

\section{Discussion}

XGP is a rare condition with an approximate incidence of 0.8 $1 \%$ of all cases of pyelonephritis. It has a moderate to strong female predisposition. ${ }^{1,3,4}$ XGP usually affects patients between the ages of 45-55 years, ${ }^{1,5}$ but has also been identified in children as young as one year old. ${ }^{1}$ There are two common cited causes of XGP, renal calculi, specifically staghorn calculi are commonly implicated. ${ }^{1,4}$ Urinary tract infection, with organisms frequently associated being Escherichia coli, Proteus mirabillus and Klebsiella species are also associated. ${ }^{1-5}$ Histologically, XGP typically leads to the destruction and replacement of the affected renal parenchyma with lipid-filled macrophages known as foam cells ${ }^{1,2}$ (Figure 1). 


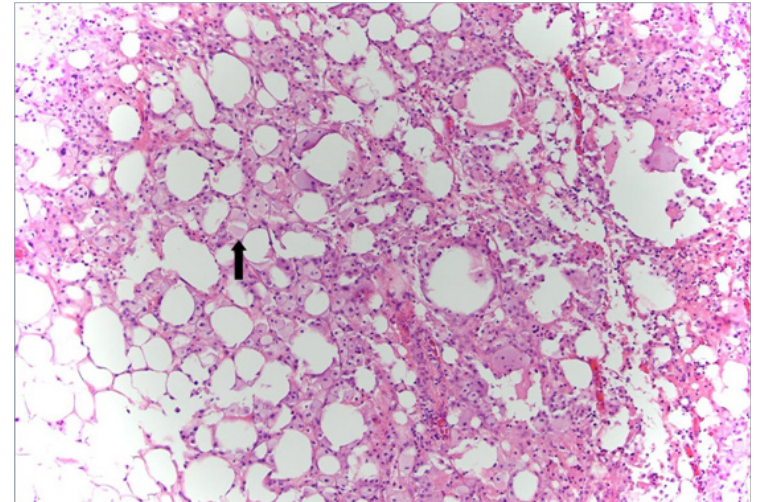

Figure I Frozen section of patient pelvic-ureteric junction demonstrating xanthogranuloma, arrow indicating foam cells.

Typically, it presents non-specifically and similar to pyelonephritis, with flank pain, fever, haematuria and lower urinary tract symptoms. ${ }^{1-3}$ XGP rarely affects both kidneys. ${ }^{4,5}$ As in this case, a significant portion of patients have malignancy considered in the differential. ${ }^{1,3,5}$ The optimal diagnostic modality has been cited as computed tomography (CT) ${ }^{1,3}$ Its advantages are that it can isolate significant disease in the majority of cases and also demonstrate associated findings such as hydronephrosis, renal parenchymal collections and abscess ${ }^{1,2}$ (Figure 2)

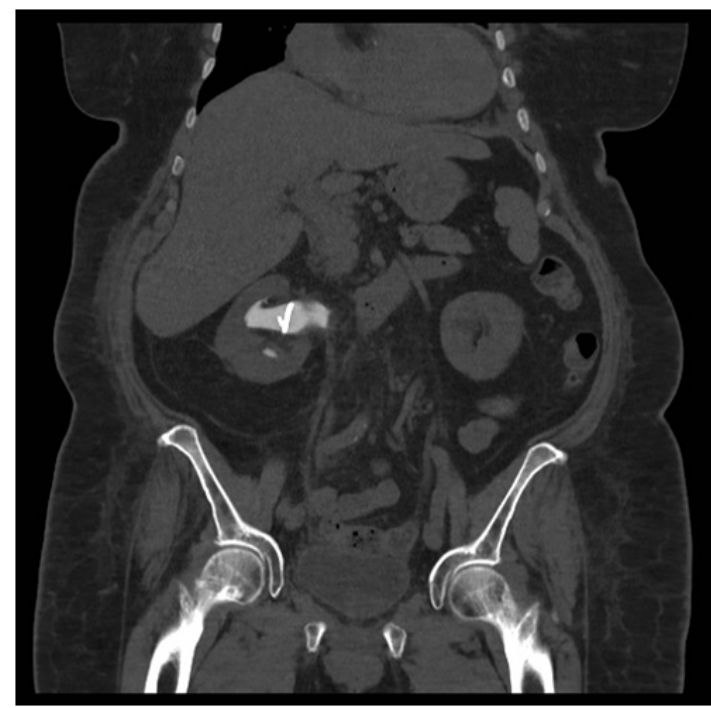

Figure 2 CT Nephrostogram demonstrating urothelial thickening.
The progression of XGP can be divided into three stages, stage 1 , where inflammation is confined to the kidney. Perinephric fat is affected in stage two and finally in stage three, the inflammation affects retroperitoneum. ${ }^{2,4}$ This case reflects the initiation of the pathophysiological process at the pelviureteric junction. The treatment of advanced XGP is frequently cited as nephrectomy, ${ }^{1,2,5}$ although cases where antibiotics have been successful have been cited. ${ }^{1,2,5}$ Surgical success can also been improved with pre-operative and post-operative antibiotic use ${ }^{1,5}$ The prognosis, with treatment of XGP is usually favourable, with rare cases of mortality associated with operative complication, such as sepsis ${ }^{1,3}$ (Figure 3 ).

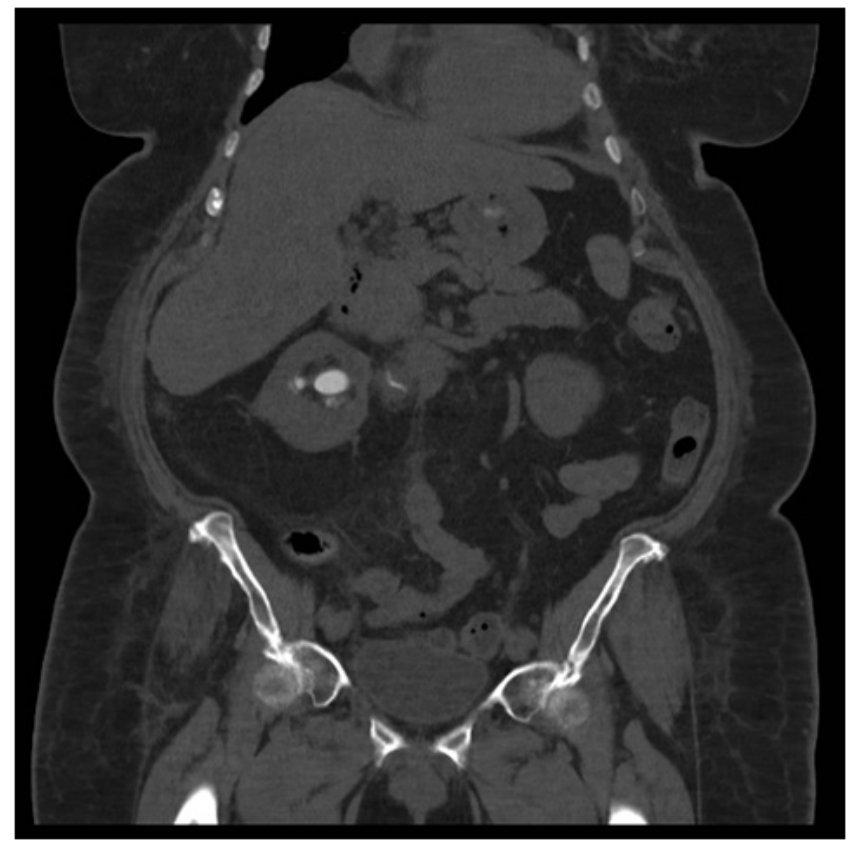

Figure 3 CT Nephrostogram demonstrating stricturing of the PUJ.

\section{Conclusion}

Xanthogranulomatous Pyelonephritis (XGP) is a rare disorder that affects mostly females; it can present similarly to calculus or infective pyelonephritis. It is best diagnosed by computed tomography and treated by nephrectomy with/without antibiotics. In the aforementioned case study, we believe, based on clinical, histological and radiological modalities that this reflects the early pathophysiology of XGP and may provide insight into the pathological process of this condition. 